\title{
Rapid inversion in shallow water with a single receiver using modal time-frequency pattern extraction
}

\author{
Julien Bonnel \\ GIPSA-Lab / DIS \\ Grenoble INP, France \\ Email: julien.bonnel@gipsa-lab.inpg.fr
}

\author{
Barbara Nicolas \\ Jérôme I. Mars \\ GIPSA-Lab / DIS \\ Grenoble INP, France
}

\author{
Dominique Fattaccioli \\ Centre Technique des Systèmes Naval \\ DGA (Toulon), France
}

\begin{abstract}
This paper presents a new inversion method in shallow water $(0-400 \mathrm{~m})$ for impulsive low-frequency source $(0$ $200 \mathrm{~Hz}$ ) using a single static receiver. In this configuration, propagation is described by modal theory. The recorded pressure field can be decomposed into modes whose arrival times (which are frequency dependant) contain information about environment. However, modes share a common frequency band; and when the radial distance between source and receiver is smaller than $15 \mathrm{~km}$, modes are also overlapped in time on the receiver. In this case, adaptive signal processing is required to identify them. First, modal propagation is quickly reviewed. Secondly, it is shown that environment information is embedded in the time-frequency structure of the modes. Then, a new inversion algorithm -simple and computationally light- using the modal arrival times is presented. Finally, it is applied on noisy simulated data.
\end{abstract}

\section{INTRODUCTION}

Environment monitoring is an important topic in oceanic engineering. It is often realized using an array of receivers and/or an array of sources. These configurations give precise results as the high number of hydrophones increases the amount of available information. However, in real experiments, positioning arrays is expensive and time consuming. To have an easier at sea configuration, some studies propose environment estimation using a single receiver. The advantage of this configuration in term of practical setting up is evident, but it drastically reduce the amount of available information to realize the inversion. As no distance aperture is available, diversity is found by using broadband source [1] [2] [3].

This paper presents a new inversion scheme in shallow water. It requires a single low-frequency broadband source and a single receiver, both static. Shallow water environment is classical in underwater acoustic, but it is still a challenging one when coupled with single receiver configuration. Indeed, it is dispersive for relatively low frequencies. The most suitable model to describe this propagation is then normal mode theory [4]. The received pressure field can be decomposed into several modes, and each mode is differently affected by dispersion: the modal arrival times differ for each mode and each frequencies. By characterizing this phenomenon, it is possible to extract information about environment and build an inversion scheme.
The inversion scheme presented in this article is based on modal arrival times analysis. It differs from the literature as it does not require any a priori information on the environment (nor for the water column neither for the bottom one), and is computationally light. It matches the environment with a Pekeris waveguide, and so identifies only few environmental parameters (mean water speed, mean bottom speed and water depth).

\section{Modal PROPAGATION}

As stated in the introduction, normal mode theory is the most suitable propagation model for shallow water $(0-400 \mathrm{~m})$ and low frequencies $(0-200 \mathrm{~Hz})$. It is quickly reviewed in this section to introduce notions and equations useful for the inversion scheme.

In a range independent environment, for an impulsive source at depth $z_{s}$ and a receiver at depth $z_{r}$ separated by a radial distance $r$, the spectrum $Y(f)$ of the received signal $y(t)$ is [4] :

$$
Y(f) \approx Q \sum_{m=1}^{N} \Psi_{m}\left(f, z_{s}\right) \Psi_{m}\left(f, z_{r}\right) \frac{e^{j k_{r m}(f) r}}{\sqrt{k_{r m}(f) r}}
$$

where $N$ is the number of modes, $\Psi_{m}$ is the modal function of mode $m$ (which is a real function depending on frequency $f$ and on depth $z$, but only values for $z_{s}$ and $z_{r}$ are taken into account), $k_{r m}(f)$ the radial wavenumber of mode $m$ (which is supposed to be real as the evanescent modes are not taken in account), and $Q=\frac{e^{j \pi / 4}}{\sqrt{8 \pi} \rho\left(z_{s}\right)}$ (with $\rho\left(z_{s}\right)$ the water density at the source depth). The influence of the environment is notably embedded into the wavenumbers $k_{r m}$.

Each component of the sum in equation 1 represents the contribution of a given mode. For a given mode $m$ (i.e. a component in equation 1), group speed $v_{g m}$ is defined by :

$$
v_{g m}(f)=2 \pi \frac{\partial f}{\partial k_{r m}}
$$

For mode number $m, v_{g m}(f)$ describes the propagation speed of frequency $f$. Group speed is a usefull notion which 
will be used in section III to define the time-frequency structure of the received signal.

\section{TIME-FREQUENCY PATTERN OF THE MODES}

Equation 1 shows that the phase of component $m$ (i.e. the phase of mode $m$ spectrum) is $k_{r m}(f) r$ (or $\pi k_{r m}(f) r$ if $\Psi_{m}\left(f, z_{s}\right) \Psi_{m}\left(f, z_{r}\right)$ is negative). Yet for a given signal, the phase of its spectrum gives the localization of this signal in the time-frequency plan. Consequently, in our case, the localization in the time-frequency plan of mode $m$ depends on $k_{r m}$. Reciprocally, if the localization in the time-frequency plan of a mode is known, information on the wavenumbers should be available, and therefore information about the environment. This is why we introduce time-frequency structure (TFS) of the received signal.

The TFS of the received signal is a sparse time-frequency representation. It is built by considering that for mode number $m$, frequency $f$ travels at speed $v_{g m}(f)$. Consequently, the TFS after modal propagation is:

$$
T F S(t, f)=\sum_{m=1}^{N} \alpha\left(m, f, r, z_{s}, z_{r}\right) \delta\left(t-\frac{r}{v_{g m}(f)}\right)
$$

where $\delta(t)$ is the Dirac distribution describing the localization of the time-frequencies structures (see Figure 1) and $\alpha$ is the attenuation term describing their amplitude. The quantity $\frac{r}{v_{g m}(f)}$ can be defined as the modal arrival time $t_{m}(f)$ of the frequency $f$ for the mode $m$ :

$$
t_{m}(f)=\frac{r}{v_{g m}(f)}
$$

These arrival times carry information about the environment as group speeds are linked to wavenumbers by equation 2 . Figure 1 presents a theoretical example of modal arrival times for a Pekeris waveguide. Note that the extreme lower frequency part of the modes (between the cutoff frequency and the Airy phase [4]) is not represented as there is almost no energy in this frequency band.

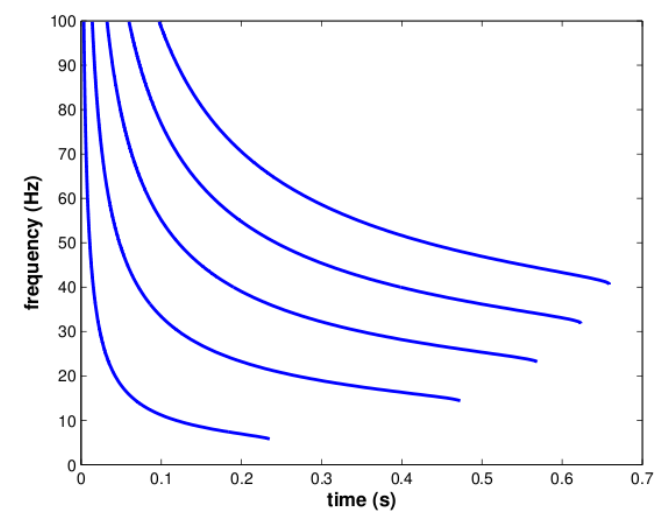

Fig. 1. Theoretical time-frequency representation of the received signal in a Pekeris waveguide

\section{INVERSION ALGORITHM}

The main idea of the algorithm is to analyze the dispersive behavior of the waveguide and use it to perform inversion. First, modal arrival times are estimated. Then they are compared with precomputed ones in order to find a good match and consequently the estimated environmental parameters.

\section{A. Estimation of the modal arrival times}

Figure 1 shows that for a given reception time several modes exist and that the received modes share a common frequency band. However, they are still separated in time-frequency domain. This is why the modal arrival times estimation is done in the time-frequency domain. Each theoretical mode presented in Figure 1 is non-stationary and has a curved structure. Classical time-frequency representations are not adapted for this kind of signal because of their inherent limitations (timefrequency uncertainty). Adaptive signal processing is required to extract modal arrival times from the received signal. In the litterature, these arrival times are often manually extracted [2] [5] or estimated using recursive algorithm [6].

This modal arrival times estimation proposed in this paper is automatic and non recursive. It will be presented in details in [7]. It is done using the following scheme:

1) Detection of the usefull part of the received signal is done using frequency warping as presented in [8]. This provides the arrival time corresponding to the direct path propagation and allows time-warping processing for the next step.

2) Modal filtering is done using time-warping as presented in [9]. The contribution of each mode in the received signal is now separated from the other.

3) For a given filtered mode, arrival times are estimated on its reallocated spectrogram [10] by ridge analysis.

The estimated arrival times are linked to the group speed by equation 4. Group speed are link to environment by equation 2 and by the following equation giving group speed from perturbation theory [4]:

$$
\frac{d k_{r m}}{d f}=4 \pi^{2} \frac{f}{k_{r m}} \int_{0}^{D} \frac{\Psi_{0 m}^{2}(z)}{\rho(z) c^{2}(z)} d z
$$

where $z$ is the depth in the water column, $f$ the frequency, $k_{r m}$ the wavenumber of mode number $m, \rho(z)$ the density at depth $z, c(z)$ the sound speed at depth $z$ and $\Psi_{0 m}(z)$ is the first order term for the modal eigenfunction $\Psi_{m}(z)$ at depth $z$. Consequently, estimated modal arrival times are a revelant characteristic of propagation and can be used in an inversion scheme.

\section{B. The inversion itself}

The inversion proposed in this section compares estimated arrival times with simulated ones. It is voluntary simple as it estimates only three geoacoustical parameters: the water celerity, the bottom celerity and the waveguide depth: the best match between the unknown environment and a Pekeris waveguide is sought. On the other hand, the algorithm is 
computationally light and no a priori information on the environment is required (neither water column nor sediment properties). Then, the algorithm process is as follow:

1) Estimate the modal arrival times

2) Find the best match between the estimated modal arrival times and precomputed ones for different values of the searched parameters.

The first step is described in section IV-A. The second step is a least mean square comparison over modes and frequency between estimated modal arrival times and simulated ones. If $\widehat{t_{m}}(f)$ is the estimated arrival time of frequency $f$ for the mode $m$ and $t_{m}^{c_{1}, c_{2}, D}(f)$ is the precomputed arrival time of frequency $f$ for the mode $m$ in a Pekeris waveguide with constant water celerity $c_{1}$, bottom celerity $c_{2}$ and water depth $D$, then the match is computed with :

$$
\begin{gathered}
{\left[\widehat{c_{1}}, \widehat{c_{2}}, \widehat{D}\right]=\underset{c_{1}, c_{2}, D}{\operatorname{Argmin}} \sum_{m} \sum_{f}\left[\widehat{t_{m}}(f)-t_{m}^{c_{1}, c_{2}, D}(f)\right]^{2}} \\
\text { V. APPLICATION }
\end{gathered}
$$

\section{A. Simulated modal arrival times}

First, the inversion algorithm is applied on simulated modal arrival times to study the cost function itself, without considering the modal arrival time estimation. The environment is a Pekeris waveguide with water sound speed $c_{1}=1500 \mathrm{~m} . \mathrm{s}^{-1}$, bottom sound speed $c_{2}=2000 \mathrm{~m} \cdot \mathrm{s}^{-1}$ and water depth $D=130 \mathrm{~m}$. The search space is:

- $c_{1} \in[1450,1550] \mathrm{m} . \mathrm{s}^{-1}$, with $5 \mathrm{~m} . \mathrm{s}^{-1}$ step

- $c_{2} \in[1900,2100] \mathrm{m} . \mathrm{s}^{-1}$, with $10 \mathrm{~m} \cdot \mathrm{s}^{-1}$ step

- $D \in[100,150] \mathrm{m}$, with $5 \mathrm{~m}$ step

As simulated arrival times are matched on simulated ones, the estimation results $\widehat{c_{1}}, \widehat{c_{2}}$ and $\widehat{D}$ are obviously the good ones. However, it is interesting to look at the shape of the cost function. Figure 2 shows the cost function in $\mathrm{dB}$ for slice at $D=130 \mathrm{~m}$, Figure 3 presents the same cost function at slice $c_{1}=1500 \mathrm{~m} \cdot \mathrm{s}^{-1}$ and Figure 4 shows it at slice $c_{2}=2000 \mathrm{~m} \cdot \mathrm{s}^{-1}$. It presents a nice gradient going to a single minimum without any ambiguous side lobes. This is good first results showing that the algorithm should be robust, even when applied on more complicated data. Moreover, Figures 3 and 4 present a sharp hole around the estimated depth, which proves that the algorithm is really depth sensitive.

\section{B. Noisy Pekeris waveguide}

To test the algorithm in a more realistic situation, the environment is now a noisy Pekeris waveguide with parameters corresponding to realistic at sea situation in shallow water:

- Water column: sound speed $c_{1}=1500 \mathrm{~m} \cdot \mathrm{s}^{-1}$, density $\rho_{1}=1000 \mathrm{~kg} \cdot \mathrm{m}^{-3}$, depth $D=130 \mathrm{~m}$

- Bottom: sound speed $c_{2}=2000 \mathrm{~m} \cdot \mathrm{s}^{-1}$, density $\rho_{2}=$ $2500 \mathrm{~kg} \cdot \mathrm{m}^{-3}$

- Depth of the source $z_{s}=20 \mathrm{~m}$

- Receiver on the bottom $z_{r}=130 \mathrm{~m}$

- Distance between source and receiver $r=4000 \mathrm{~m}$

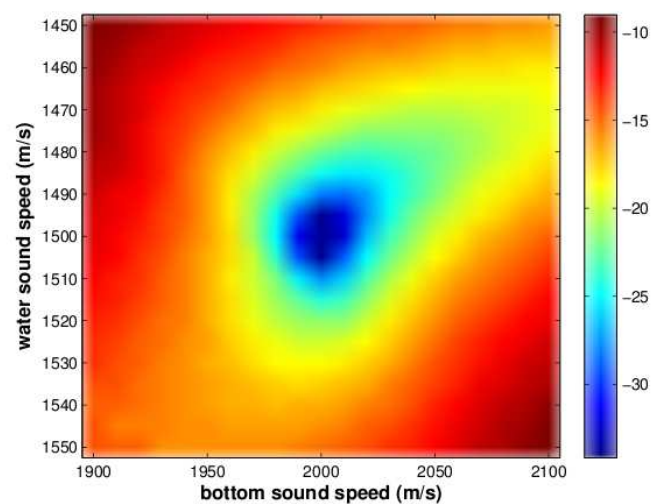

Fig. 2. Cost function in $d B$ for simulated arrival times, slice at $D=130 \mathrm{~m}$

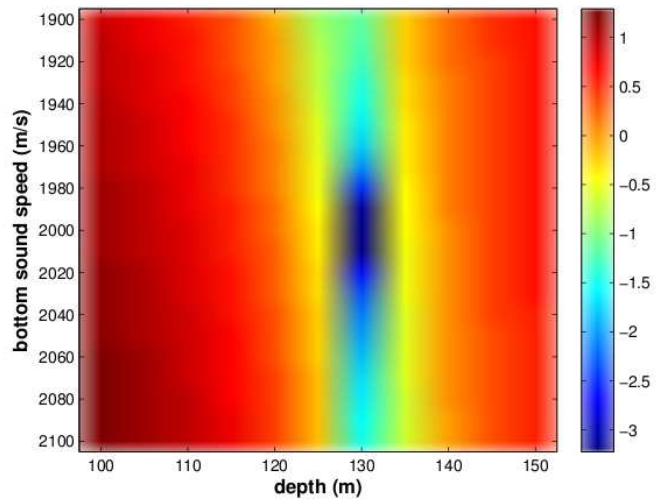

Fig. 3. Cost function in $\mathrm{dB}$ for simulated arrival times, slice at $\mathrm{c} 1=1500 \mathrm{~m} / \mathrm{s}$

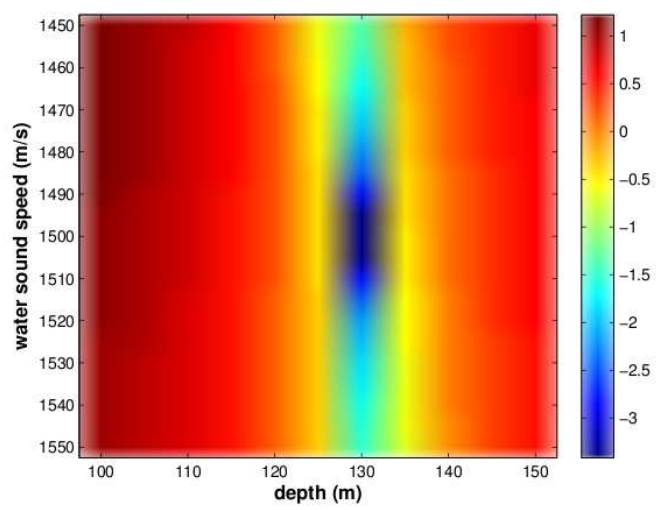

Fig. 4. Cost function in $\mathrm{dB}$ for simulated arrival times, slice at $\mathrm{c} 2=2000 \mathrm{~m} / \mathrm{s}$

- Sampling frequency $F_{s}=200 \mathrm{~Hz}$

- Signal to Noise Ratio $S N R=5 \mathrm{~dB}$ (gaussian white noise)

The simulated signal in time is presented in Figure 5 and its spectrum in Figure 6. As stated in section IV-A, modes are overlapped in the time domain and in the frequency domain and they cannot be distinguished. The spectrogram of the 
received signal is presented in Figure 7, the modal pattern can be guessed, although it is not clear because of time-frequency representations limitations. The result of the estimation of the modal arrival times is presented with the color curved lines in Figure 8. The detection has been done thanks to frequency warping so just the usefull part of the signal is presented, and the estimated arrival times are surimposed on it. Visually, the estimation of these arrival times look good. If the estimated arrival times are compared to the real ones, the mean estimation error ignoring mode 1 is $5.2 \mathrm{~ms}$ (as sampling frequency is $200 \mathrm{~Hz}$, it is a single sample error). Mode 1 will not be used in the rest of the inversion scheme as it is not energetic enough and the estimation of its arrival time is not precise.

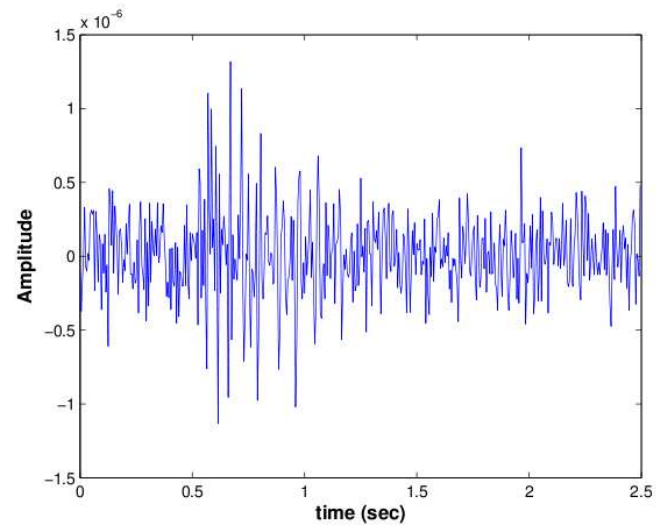

Fig. 5. Simulated signal in a Pekeris waveguide $(\mathrm{SNR}=5 \mathrm{~dB})$

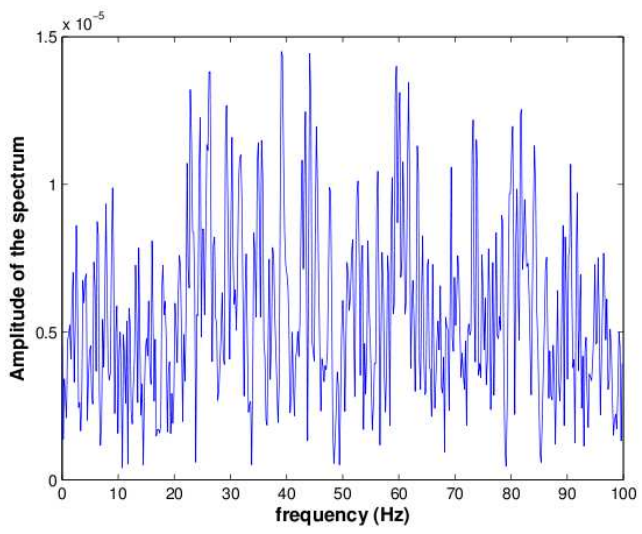

Fig. 6. Spectrum of the simulated signal in a Pekeris waveguide $(\mathrm{SNR}=5 \mathrm{~dB})$

The inversion algorithm is applied modal using arrival times for mode 2 to 5 , and the results is:

- $\widehat{c_{1}}=1500 \mathrm{~m} \cdot \mathrm{s}^{-1}$

- $\widehat{c_{2}}=1970 \mathrm{~m} \cdot \mathrm{s}^{-1}$

- $\widehat{D}=130 \mathrm{~m}$

These results are really good. As expected, the depth is perfectly estimated. The water sound speed is also perfectly estimated, and there is only a slightly error (1.5\%) for the

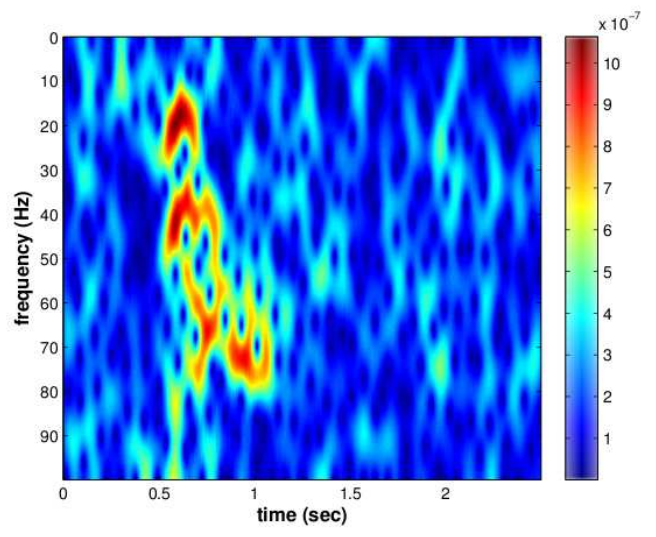

Fig. 7. Spectrogram of the simulated signal in a Pekeris waveguide $(\mathrm{SNR}=5 \mathrm{~dB})$

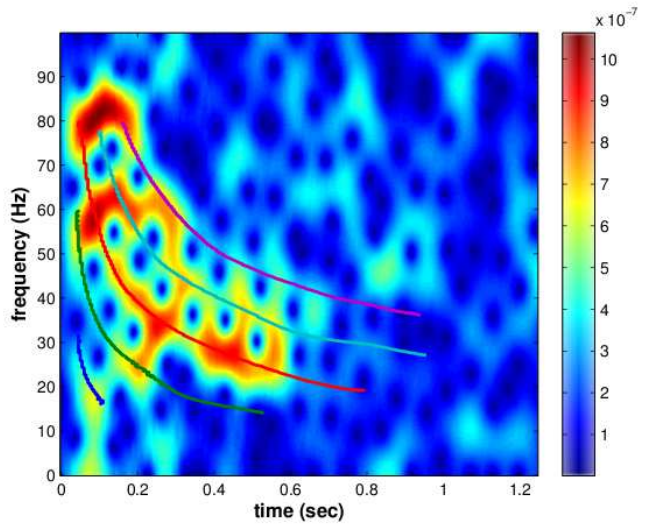

Fig. 8. Result of modal arrival times estimation for the Pekeris waveguide $(\mathrm{SNR}=5 \mathrm{~dB})$

bottom sound speed estimation. Slices of the cost function at $D=130 \mathrm{~m}$, at $c_{1}=1500 \mathrm{~m} \cdot \mathrm{s}^{-1}$ and at $c_{2}=2000 \mathrm{~m} \cdot \mathrm{s}^{-1}$ are respectively presented in Figures 9, 10 and 11. It can be seen that even in this more realistic case, there is no local minimum which can create ambiguity for the results. Inversion quality was not decreased by modal arrival time estimation.

Using the estimated values $\widehat{c_{1}}, \widehat{c_{2}}$ and $\widehat{D}$, wavenumbers were computed and compared to wavenumbers corresponding to the real environment. Figure 12 presents the mean error over modes 1 to 5 as a function of frequency for the estimated wavenumbers. The mean error over frequency and over modes 1 to 5 is $0.06 \%$. Consequently, the inversion algorithm gives really good results on noisy simulations, and this is promising before applying it on real data.

\section{CONCLUSION}

Impulsive low frequency underwater signals $(<200 \mathrm{~Hz})$ can be natural (marine mammals) or man-made (air gun). For shallow water $(<400 \mathrm{~m})$, the propagation in this frequency range is described by normal mode theory: the pressure field can be decomposed into several modes. As the medium is dispersive, the propagation speed of a given mode depends 


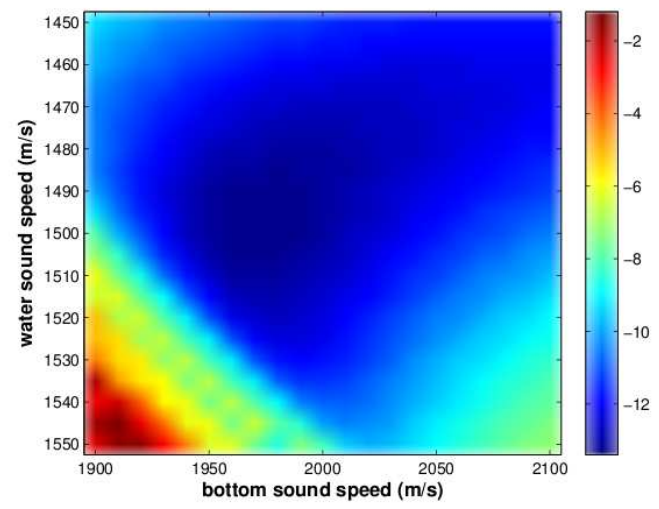

Fig. 9. Cost function in $\mathrm{dB}$ for the Pekeris waveguide $(\mathrm{SNR}=5 \mathrm{~dB})$, slice at $\mathrm{D}=130 \mathrm{~m}$

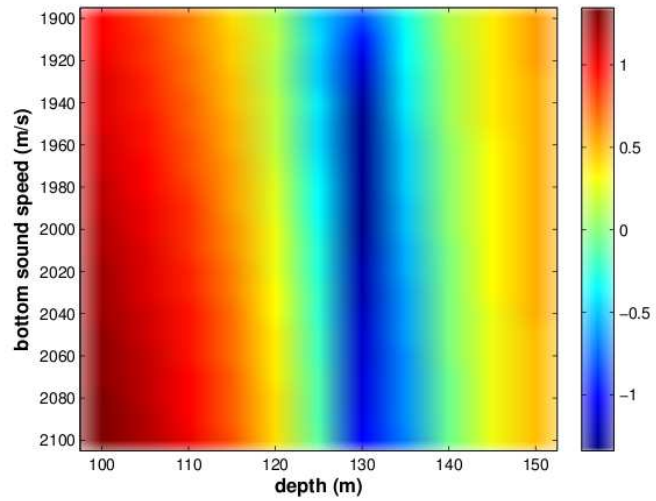

Fig. 10. Cost function in $\mathrm{dB}$ for the Pekeris waveguide $(\mathrm{SNR}=5 \mathrm{~dB})$, slice at $\mathrm{c} 1=1500 \mathrm{~m} / \mathrm{s}$

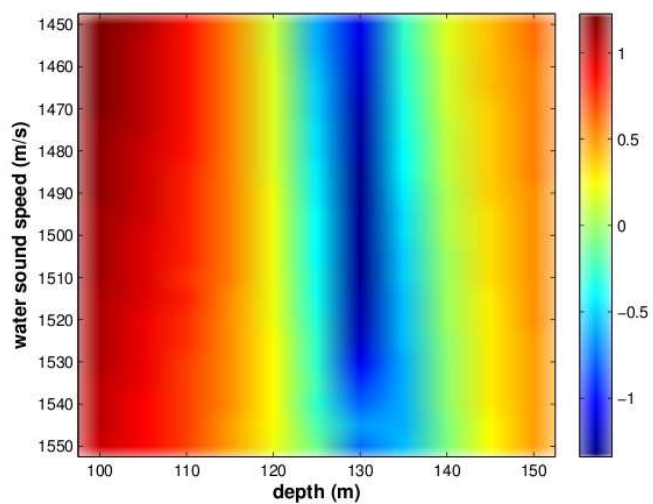

Fig. 11. Cost function in $\mathrm{dB}$ for the Pekeris waveguide $(\mathrm{SNR}=5 \mathrm{~dB})$, slice at $\mathrm{c} 2=2000 \mathrm{~m} / \mathrm{s}$

on frequency: modal arrival times depending of frequency can be defined and estimated using a single receiver. This article shows that these modal arrival times are a good feature of the propagation and can be used in an inversion scheme. The algorithm presented is simple as it estimates only three waveguides parameters: the water celerity, the bottom celerity

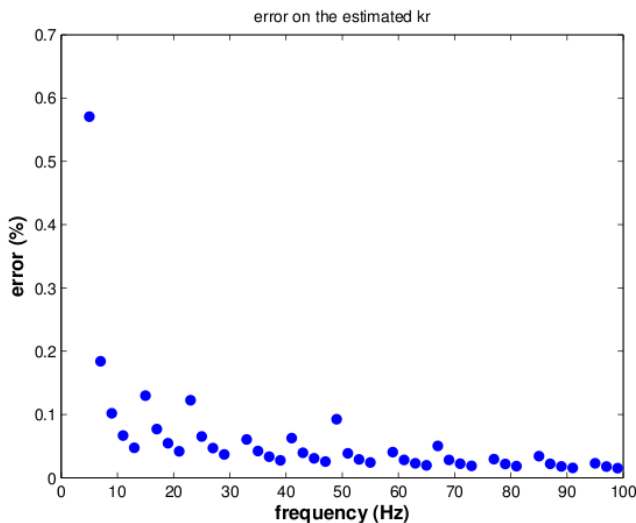

Fig. 12. Error on the estimated wavenumbers after inversion in the Pekeris waveguide $(\mathrm{SNR}=5 \mathrm{~dB})$

and the waveguide depth. However, it is computationally light and does not require any a priori information on the environment. It gives good result on noisy simulated data. As a perspective, the authors would like to apply the algorithm on real data.

\section{ACKNOWLEDGMENT}

The authors would like to thank W.A. Kuperman, S. Walker and B. Cornuelle from Marine Physical Laboratory (Scripps Institution of Oceanography) for helpful discussions, DGA for founding this research, and Explora'Doc for founding displacements between GIPSA-Lab (Grenoble-INP, France) and Marine Physical Laboratory (Scripps Institution of Oceanography, USA).

\section{REFERENCES}

[1] J.P. Hermand. Broad-band geoacoustic inversion in shallow water from waveguideimpulse response measurements on a single hydrophone: theory and experimental results. IEEE Journal of Oceanic engineering, vol.24, no.1, pp. 41-66, 1999.

[2] G.R. Potty, J.H. Miller, J.F. Lynch, K.B. Smith. Tomographic inversion for sediment parameters in shallow water. The Journal of the Acoustical Society of America, vol.108, no.3, pp. 973-986, 2000.

[3] J.C. Le Gac, M. Asch, Y. Stephan, X. Demoulin. Geoacoustic inversion of broad-band acoustic data in shallow water on a single hydrophone IEEE Journal of Oceanic engineering, vol.28, no.3, pp. 479-493, 2003.

[4] F.B. Jensen. Computational ocean acoustics. Amer Inst of Physics, 1994.

[5] Y. Stephan, Y. Simard, C. Gevaise, S. Vallez. Robust 2D localization of low-frequency calls in shallow waters using modal propagation modelling. Canadian Acoustics, vol.36, no.1, pp.153-159, 2008.

[6] J.C. Hong, K.H. Sun, Y.Y. Kim. Dispersion-based short-time Fourier transform applied to dispersive wave analysis. The Journal of the Acoustical Society of America, vol.117, no.5, pp.2949-2960, 2005.

[7] J. Bonnel, B. Nicolas, J.I. Mars, S.C. Walker. Estimation of modal arrival times with a single receiver for geoacoustic inversion in shallow water. IEEE Journal of Oceanic engineering, to be submited.

[8] J. Bonnel, B. Nicolas, J.I. Mars, G. Le Touzé, D. Fattaccioli Mode characterization in shallow water using warping tools. The Journal of the Acoustical Society of America, vol.125, pp.2541, 2009.

[9] J. Bonnel, G. Le Touzé, B. Nicolas, J.I. Mars, C. Gervaise. Automatic and passive whale localization in shallow water using gunshots. Oceans'08 MTS/IEEE, Quebec city, 2008.

[10] F. Auger, P. Flandrin, Improving the Readability of Time-Frequency and Time-Scale Representations by the Reassignment Method. IEEE Transactions on Signal Processing, vol.43, no.5, pp.1068-1089, 1995 